\title{
Commercial Laying Hen Diets Formulated According to Different Recommendations of Total and Digestible Amino Acids
}

\section{author(s) \\ Casartelli $\mathrm{EM}^{*}$ \\ Filardi RS \\ Junqueira $\mathrm{OM}$ \\ Laurentiz AC \\ Assuena $\mathrm{V}$ \\ Duarte KF}

Departamento de Zootecnia, Universidade Estadual Paulista, Faculdade de Ciências Agrárias e Veterinárias, Campus de Jaboticabal, via de acesso Prof. Dr. Paulo Donato Castellane, s/n, Jaboticabal, São Paulo, Brasil.

\section{Mail Address}

Elena M Casartelli

Av. Líbero Badaró, 810

Centro

14.870-540. Jaboticabal, SP, Brazil.

E-mail: emcasartelli@yahoo.com.br

\section{Keywords}

Egg quality, layers, nutrition, performance, poultry.

\section{ABSTRACT}

An experiment was conducted to evaluate different commercial laying hen diets formulated based on recommendations for total and digestible amino acids. One hundred and twenty Lohmann LSL commercial laying hens aged 25 weeks were distributed in a completely randomized experimental design involving five replications of six birds in four treatments. Diet formulation on a total amino acid basis followed the recommendations of NRC (1994) and Rostagno et al. (2000), whereas formulation on digestible amino acids basis was according to Rostagno et al. (2000) and Degussa (1997) recommendations. The experimental period was divided into five periods of fourteen days. Performance parameters (egg production, feed intake, feed conversion, egg mass) were evaluated for each period, and on the last two days of each period, three eggs per replication were collected to evaluate egg quality parameters (Haugh unit, egg specific gravity, egg weight, eggshell thickness and percentage). Means were compared by orthogonal contrasts. Results on feed intake, egg production, egg mass, feed conversion and egg specific gravity showed that total amino acid recommendations promoted better bird responses than digestible amino acid recommendations.

\section{INTRODUCTION}

In spite of the well known importance of the amino acid composition of ingredients used for diet formulation, this knowledge is restricted due to the little information on digestible amino acids compared to the information on total amino acids in the majority of the ingredients that are used in animal feeding.

The low amino acid digestibility of an ingredient might be related to anti-nutritional factors, processing, physical or chemical traits or to high fiber levels (Parsons, 1992). Processing parameters, such as high temperature or pressure, might contribute significantly to the reduction of amino acid availability, especially in ingredients as sub-products of cereal, oilseed or animal by-product industry. Thus, when diets are formulated using such ingredients, the contents of digestible amino acids should be considered instead of total amino acid levels, in order to reduce mistakes in amino acid supplementation in the diet.

The benefits of diet formulation based on digestible amino acids and ideal protein have been demonstrated in many trials with broiler chickens (Rostagno et al., 1995; Wang \& Parsons, 1998; Douglas \& Parsons, 1999; Araújo, 2001). On the other hand, there are not enough studies with commercial laying hens. Therefore, the objective of this study was to evaluate different recommendations of total (NRC, 1994, and Rostagno et al., 2000) and digestible (Rostagno et al., 2000 and Degussa, 1997) amino acids on performance and egg quality of commercial laying hens. 


\section{MATERIAL AND METHODS}

The experiment was conducted at the Experimental Poultry Farm from Faculdade de Ciências Agrárias e Veterinárias (FCAV-UNESP), Jaboticabal, State of São Paulo, Brazil, during five periods of fourteen days.

A total of one hundred and twenty commercial Lohmann LSL layers at 25 weeks of age were distributed in a completely randomized experimental design with five replications of six birds each. Treatments were based on four different recommendations of amino acids; two diets were formulated to fulfill the requirements of total amino acid (NRC, 1994 plus 10\% and Rostagno et al., 2000) and two formulated on a digestible amino acid basis (Rostagno et al., 2000 and Degussa, 1997), described as follows:

- TA - NRC - Diet based on total amino acid as recommended by NRC (1994) plus10\%;

- TA - ROS - Diet based on total amino acid as recommended by Rostagno et al. (2000);

- DA - ROS - Diet based on digestible amino acid as recommended by Rostagno et al. (2000);

- DA - DEG - Diet based on digestible amino acid as recommended by Degussa (1997).

All diets had the same levels of energy (2.85 Mcal $\mathrm{ME} / \mathrm{kg}$ ), calcium (3.65\% Ca) and available phosphorus $(0.34 \% \mathrm{Pd})$. Protein levels were the same within TA or DA treatments, i.e., $14.5 \% \mathrm{CP}$ and $12.8 \% \mathrm{CP}$, respectively. Percentage compositions and calculated values of the diets are presented in Table 1.

At the end of the each period, bird performance was evaluated by feed intake ( $\mathrm{Fl}-\mathrm{g} / \mathrm{bird} /$ day), egg production (EP - \%), egg mass (EM - g), egg weight (EW - g), and feed conversion (FC - kg feed/kg eggs).

The parameters relative to egg quality were evaluated during the last two days of each period. Three eggs were randomly collected per replication in order to determine eggshell thickness (ST - mm), Haugh units (HU), and eggshell percentage (SP - \%). Egg specific gravity (ESG $-\mathrm{g} / \mathrm{cm}^{3}$ ) was determined using all eggs of each replication on the evaluation days. It was used $\mathrm{NaCl}$ solutions with density ranging between 1.065 and $1.100 \mathrm{~g} / \mathrm{cm}^{3}$ at $0.005 \mathrm{~g} / \mathrm{cm}^{3}$ increments, according to the procedure described by according Moreng \& Avens (1990).

The obtained data were submitted to the analysis of variance using the General Linear Models procedure (GLM) of the SAS ${ }^{\circledR}$ software (SAS Institute, 1996). Means were compared by orthogonal contrasts as follows:
- Contrast $1 \rightarrow$ TA means vs DA means

- Contrast $2 \rightarrow$ TA - NRC means vs TA-ROS means

- Contrast $3 \rightarrow$ DA - ROS means vs DA-DEG means

\begin{tabular}{|c|c|c|c|c|}
\hline \multicolumn{5}{|c|}{$\begin{array}{l}\text { Table } 1 \text { - Percentage composition of diets and calculatec } \\
\text { nutritional levels. }\end{array}$} \\
\hline Corn & 64.18 & 64.04 & 70.24 & 69.66 \\
\hline Soybean meal & 18.33 & 18.34 & 12.78 & 13.10 \\
\hline Rice meal & 5.00 & 5.00 & 5.00 & 5.00 \\
\hline Limestone & 8.46 & 8.46 & 8.49 & 8.48 \\
\hline Dicalcium phosphate & 1.29 & 1.29 & 1.32 & 1.32 \\
\hline Soybean oil & 1.69 & 1.74 & 0.79 & 0.93 \\
\hline Sodium chloride & 0.43 & 0.43 & 0.44 & 0.44 \\
\hline DL - Methionine (98\%) & 0.08 & 0.12 & 0.18 & 0.22 \\
\hline L - Lysine $\mathrm{HCl}(78 \%)$ & 0.00 & 0.04 & 0.19 & 0.25 \\
\hline L - Threonine & 0.00 & 0.00 & 0.00 & 0.02 \\
\hline L - Tryptophan & 0.00 & 0.00 & 0.03 & 0.04 \\
\hline Antioxidant & 0.04 & 0.04 & 0.04 & 0.04 \\
\hline Vitam. Min. Suplement* & 0.50 & 0.50 & 0.50 & 0.50 \\
\hline Total & 100.00 & 100.00 & 100.00 & 100.00 \\
\hline \multicolumn{5}{|l|}{ Calculated Levels } \\
\hline Met. Energy (Mcal/kg) & 2.85 & 2.85 & 2.85 & 2.85 \\
\hline Crude protein (\%) & 14.50 & 14.50 & 12.50 & 12.50 \\
\hline Calcium (\%) & 3.65 & 3.65 & 3.65 & 3.65 \\
\hline Available phosphorus (\%) & 0.34 & 0.34 & 0.34 & 0.34 \\
\hline Total arginine (\%) & 0.91 & 0.91 & 0.75 & 0.77 \\
\hline Total lysine (\%) & 0.69 & 0.72 & 0.71 & 0.77 \\
\hline Total Met + Cys (\%) & 0.58 & 0.62 & 0.61 & 0.67 \\
\hline Total methionine (\%) & 0.33 & 0.36 & 0.39 & 0.44 \\
\hline Total threonine (\%) & 0.56 & 0.56 & 0.48 & 0.50 \\
\hline Total tryptophan (\%) & 0.16 & 0.16 & 0.16 & 0.16 \\
\hline Total leucine (\%) & 1.36 & 1.36 & 1.23 & 1.24 \\
\hline Total isoleucine (\%) & 0.60 & 0.60 & 0.50 & 0.50 \\
\hline Total valine (\%) & 0.68 & 0.68 & 0.59 & 0.59 \\
\hline Total histidine (\%) & 0.40 & 0.40 & 0.35 & 0.35 \\
\hline Digestible arginine (\%) & 0.85 & 0.85 & 0.70 & 0.71 \\
\hline Digestible lysine (\%) & 0.61 & 0.63 & 0.64 & 0.71 \\
\hline Digestible Met +Cys (\%) & 0.52 & 0.55 & 0.56 & 0.62 \\
\hline Digestible methionine (\%) & 0.30 & 0.34 & 0.37 & 0.42 \\
\hline Digestible threonine (\%) & 0.48 & 0.48 & 0.40 & 0.44 \\
\hline Digestible tryptophan (\%) & 0.15 & 0.15 & 0.15 & 0.15 \\
\hline Digestible leucine (\%) & 1.22 & 1.22 & 1.11 & 1.12 \\
\hline Digestible isoleucine (\%) & 0.52 & 0.52 & 0.43 & 0.44 \\
\hline Digestible valine (\%) & 0.59 & 0.59 & 0.50 & 0.51 \\
\hline Digestible histidine (\%) & 0.35 & 0.35 & 0.30 & 0.30 \\
\hline Sodium (\%) & 0.20 & 0.20 & 0.20 & 0.20 \\
\hline
\end{tabular}

* Values added per $\mathrm{kg}$ of diet: Vit A - 8,000 IU; Vit D3 - 2,000 IU; Vit E - 50 mg; Vit K3 - 3 mg; Vit B1 - 1.5 mg; Vit B2 - 4 mg; Vit B6 - 0.12 $\mathrm{mg}$. Vit B12 - $15 \mathrm{mcg}$; Folacin - $0.6 \mathrm{mg}$; Pantothenic Acid $10 \mathrm{mg}$; Niacin - 30 mg; Biotin - 0.1 mg; Choline - 300 mg; Iron - 50 mg; Copper - 10 mg; Zinc - 70 mg; Manganese - 100 mg; lodine - 1 mg; Selenium $-0.3 \mathrm{mg}$; Antioxidant $50 \mathrm{mg}$.

\section{RESULTS}

Analyzing performance results (Table 2), it was observed that feed intake, egg production, egg mass and feed conversion of contrast 1 were significantly different $(p<0.01)$. Hens fed diets formulated based on total amino acid basis had better performance that 
Casartelli EM, Filardi RS, Junqueira

OM, Laurentiz AC, Assuena V, Duarte KT those fed diets formulated based on digestible amino acid basis. Considering the same parameters, there were no significant differences between NRC (1994) and Rostagno et al. (2000) recommendations for total amino acid requirements or between Rostagno et al. (2000) and Degussa (1997) for digestible amino acids. Diets formulated on digestible amino acid basis according to Rostagno et al. (2000) promoted heavier eggs $(p<0.05)$ than Degussa (1997) formulation.

\begin{tabular}{|c|c|c|c|c|c|}
\hline Treatments & FI & EP & EW & EM & FC \\
\hline C1 & 22.70 ** & $60.09 * *$ & $1.99^{\mathrm{NS}}$ & $40.99 * *$ & $14.07 * *$ \\
\hline C2 & $0.71^{\mathrm{NS}}$ & $2.25^{\mathrm{NS}}$ & $0.48^{\mathrm{NS}}$ & $0.54^{\mathrm{NS}}$ & $0.00^{\mathrm{NS}}$ \\
\hline C3 & $0.24^{\mathrm{NS}}$ & $1.00^{\mathrm{NS}}$ & $8.26^{*}$ & $0.58^{\mathrm{NS}}$ & $0.02^{\mathrm{NS}}$ \\
\hline CV\% & 3.90 & 3.56 & 2.62 & 4.87 & 3.85 \\
\hline TA-NRC & 100.20 & 95.53 & 61.29 & 58.55 & 1.712 \\
\hline TA-ROS & 98.15 & 92.54 & 61.99 & 57.33 & 1.714 \\
\hline DA-ROS & 91.81 & 82.10 & 62.09 & 51.03 & 1.824 \\
\hline DA-DEG & 90.66 & 84.10 & 59.17 & 49.76 & 1.830 \\
\hline
\end{tabular}

NS $=$ non significant; ${ }^{*} p<0.05 ; * * p<0.01 . C 1$ - Contrast $1 \rightarrow$ TA means vs DA means; C2 - Contrast $2 \rightarrow$ TA - NRC means vs TA - ROS means; C3 - Contrast $3 \rightarrow$ DA - ROS means vs DA - DEG means.

Table 3 shows the results of egg quality parameters. Haugh Unity was superior $(p<0.01)$ for hens fed diets formulated according to total amino acid requirements of NRC (1994) in comparison to Rostagno et al. (2000) recommendations. Rostagno et al. (2000) recommendations resulted in higher $(p<0.05)$ shell thickness than Degussa (1997) recommendations on digestible amino acid basis.

\begin{tabular}{|c|c|c|c|c|}
\hline \multicolumn{5}{|c|}{$\begin{array}{l}\text { Table } 3 \text { - Egg quality of laying hens fed on total and digestible } \\
\text { amino acids basis. HU: Haugh unity; SP: shell percentage }(\%) \text {; } \\
\text { ST: shell thickness (mm); ESG: egg specific gravity }\left(\mathrm{g} / \mathrm{dm}^{3}\right) \text {. }\end{array}$} \\
\hline Treatments & HU & SP & ST & ESG \\
\hline C1 & $4.29^{\mathrm{NS}}$ & $0.58^{\mathrm{NS}}$ & $0.46^{\mathrm{NS}}$ & $6.22^{*}$ \\
\hline C2 & $9.38^{* *}$ & $0.12^{\mathrm{NS}}$ & $0.97^{\mathrm{NS}}$ & $0.01^{\mathrm{NS}}$ \\
\hline C3 & $1.75^{\mathrm{NS}}$ & $1.09^{\mathrm{NS}}$ & $4.77^{*}$ & $1.57^{\mathrm{NS}}$ \\
\hline CV\% & 1.02 & 2.12 & 2.71 & 0.13 \\
\hline TA-NRC & 96.60 & 9.51 & 0.388 & 1.0900 \\
\hline TA-ROS & 94.70 & 9.55 & 0.395 & 1.0900 \\
\hline DA-ROS & 96.97 & 9.67 & 0.396 & 1.0890 \\
\hline DA-DEG & 96.15 & 9.53 & 0.381 & 1.0879 \\
\hline
\end{tabular}

NS = non significant; ${ }^{*} \mathrm{p}<0.05 ; * * \mathrm{p}<0.01 . \mathrm{C} 1$ - Contrast $1 \rightarrow \mathrm{TA}$ means vs DA means; C2 - Contrast $2 \rightarrow$ TA - NRC means vs TA - ROS means; C 3 - Contrast $3 \rightarrow$ DA - ROS means vs DA - DEG means.

Total amino acid recommendations improved $(p<0.05)$ egg specific gravity compared to recommendations for digestible amino acids.
Commercial Laying Hen Diets Formulated According to Different Recommendations of Total and Digestible Amino Acids

\section{DISCUSSION}

Bird performance was worse when diets were formulated based on digestible amino acids probably because of the low crude protein levels $(12.5 \%)$ that were used in order to attend the requirements of digestible amino acids, although synthetic amino acids have been supplemented to fulfill methionine, methionine + cysteine, lysine, tryptophan and threonine requirements, the low protein level of these diets determined low levels of isoleucine and valine (around 13.02 and $5.4 \%$, respectively), which might have caused lower intake of these amino acids and the consequent deficiencies compared to the birds fed diets formulated based on total amino acid requirements.

Although some studies using heavy hens (Lopes \& Leeson, 1995) and lean laying hens (Hussein \& Harms, 1994, Camps \& Edghill, 1999) have shown the possibility of reducing the diet protein level when synthetic amino acids are supplemented, the present findings suggest that crude protein levels of $12.5 \%$ might reduce nitrogen availability to be used in the synthesis of nonessential amino acids. According to Keshavarz \& Jackson (1992), essential amino acids might be converted to non-essential amino acids, reducing the availability of the former and, consequently, reducing bird performance.

Total or digestible amino acid recommendations did not produce marked differences in egg quality parameters but they had significant effects on performance traits. The crude protein level of $12.5 \%$ might have had a significant effect on the reduction of performance. However, the reduction in crude protein levels from $14.5 \%$ (total amino acids recommendations) to $12.5 \%$ (digestible amino acids recommendations), considering the supplementation with methionine, methionine+cysteine, lysine, tryptophan and threonine, did not affect egg quality. Similarly, Camps \& Edghill (1999) observed that the reduction in crude protein from $16 \%$ to $12 \%$ CP in laying hen diets affected negatively bird performance, whereas no negative effects were seen on egg quality evaluated at 40 weeks of age due to the lower protein level.

\section{CONCLUSION}

In conclusion, total amino acid recommendations resulted in better performance and higher egg specific gravity in relation to digestible amino acid recommendations. However, there were no 
differences between the two diets formulated according to total amino acid recommendations and between the two diets formulated on digestible amino acid basis.

\section{REFERENCES}

Araújo LF. Estudo de diferentes critérios de formulação de rações com base em perfis de aminoácidos totais e digestíveis para frangos de corte [tese]. Jaboticabal (SP):Universidade Estadual Paulista; 2001.

Camps DM, Edghill E. Niveles de proteina en dietas para ponedoras. Revista Cubana de Ciencia Avícola 1999; 23(1):35-40.

Degussa. Amino acid requirements for poultry. Feed formulation guide. Hanau: Degussa AG; 1997

Douglas MW, Parsons CM. Dietary formulation with rendered spent hen meals on a total amino acid versus a digestible amino acid basis. Poultry Science 1999; 78(4):556-560.

Hussein SM, Harms RH. Effect of amino acid deficiencies on yolk:albumen ratio in hen eggs. Journal Applied Poultry Research 1994; 3(4):362-366.

Keshavarz K, Jackson ME. Performance of growing pullets and laying hens fed low-protein, amino acid supplemented diets. Poultry Science 1992; 71(5):905-918.

Lopes G, Leeson S. Response of broiler breeders to low-protein diets. 1. Adult breeder performance. Poultry Science 1995; 74(4): 685-695.

Moreng RE, Avens JS. Ciência e produção de aves. São Paulo: Roca; 1990. 380 p.

National Research Council. Nutrient requirements of poultry. $9^{\text {th }}$ ed. Washington: National Academic Press; 1994.

Parsons C.M. Application of the concept of amino acid availability in practical feed formulation. In: International Technical Symposia; 1992; Curaçao. Proceedings... Curaçao: Novus International; 1992. p. 43-50.

Rostagno HS, Pupa JMR, Pack M. Diet formulation for broilers based on total versus digestible amino acid. Journal Applied Poultry Research 1995; 4(3):293-299.

Rostagno HS, Albino LFT, Donzele JL, Gomes PC, Ferreira AS, Oliveira RF, Lopes DC. Composição de alimentos e exigências nutricionais de aves e suínos: tabelas brasileiras. Viçosa, MG: UFV; 2000. 141 p.

Statistical Analysis System.User's guide: statistics. Cary: SAS Institute; 1996.

Wang $X$, Parsons M. Dietary formulation with meat and bone meal on a total versus a digestible amino acid basis. Poultry Science 1998; 77(7):1010-1015. 